\title{
CONF-9709121_.
}

\section{HEAT CAPACITY OF POLY(TRIMETHYLENE TEREPHTHALATE)}

\author{
M. Pyda ${ }^{1,2}$, A. Boller ${ }^{1,2}$, J. Grebowicz ${ }^{3}$, H. Chuah ${ }^{3}$ and B. Wunderlich ${ }^{1,2 *}$ \\ ${ }^{1}$ Dept. of Chemistry, The University of Tennessee, Knoxville, TN 37996-1600 \\ ${ }^{2}$ Chem. and Anal. Sci. Div., Oak Ridge Natl. Lab., Oak Ridge, TN 37831-6197 \\ ${ }^{3}$ Shell Development Company, Houston, TX 77251-1380
}

\begin{abstract}
Thermal analysis of poly(trimethylene terephthalate) (PTT) has been carried out using standard differential scanning calorimetry and temperature-modulated differential scanning calorimetry. Heat capacities of the solid and liquid states of semicrystalline PTT are reported from $190 \mathrm{~K}$ to $570 \mathrm{~K}$. The semicrystalline PTT has a glass transition temperature of about $331 \mathrm{~K}$. Between $460 \mathrm{~K}$ and $480 \mathrm{~K}$, PTT shows an exothermic ordering. The melting endotherm occurs between $480 \mathrm{~K}$ and $505 \mathrm{~K}$ with an onset temperature of $489.15 \mathrm{~K}\left(216^{\circ} \mathrm{C}\right)$. The heat of fusion of typical semicrystalline samples is $13.8 \mathrm{~kJ} / \mathrm{mol}$. For $100 \%$ crystalline PTT the heat of fusion is estimated to be $28-30 \mathrm{~kJ} / \mathrm{mol}$. The heat capacity of solid PTT is linked to an approximate group vibrational spectrum, and the Tarasov equation is used to estimate the skeletal vibrational heat capacity $\left(\Theta_{1}=542 \mathrm{~K}\right.$ and $\left.\Theta_{3}=42 \mathrm{~K}\right)$. A comparison of calculation and experimental heat capacities show agreement of better than $\pm 2 \%$ between $190-300 \mathrm{~K}$. The experimental heat capacity of liquid PTT can be expressed as a linear function of temperature: $C_{p}{ }^{2}(\exp )=211.6+0.434 \mathrm{~T} \mathrm{~J} /(\mathrm{K}$ $\mathrm{mol}$ ) and compares well with estimations from the ATHAS data bank using group contributions of other polymers with the same constituent groups $( \pm 0.5 \%)$. The change of heat capacity at $T_{g}$ of amorphous PTT has been estimated from the heat capacities of liquid and solid to be $86.4 \mathrm{~J} /(\mathrm{K} \mathrm{mol})$. Knowing $C_{p}$ of the solid, liquid, and the transition parameters, the thermodynamic functions: enthalpy, entropy and Gibbs function were obtained. With these data, one can compute the crystallinity changes with temperature and the mobile and rigid amorphous fractions.
\end{abstract}

\section{Introduction}

Poly(trimethylene terephthalate) (PTT) is a semicrystalline, polymeric material, currently developed for fiber applications. The molecule of PTT belongs to the homologous series of aromatic polyesters with poly(ethylene terephthalate) PET and poly(butylene terephthalate) (PBT) as most important representatives. Its repeating unit, with a mass $M_{w}=206.2 \mathrm{Da}$, is:

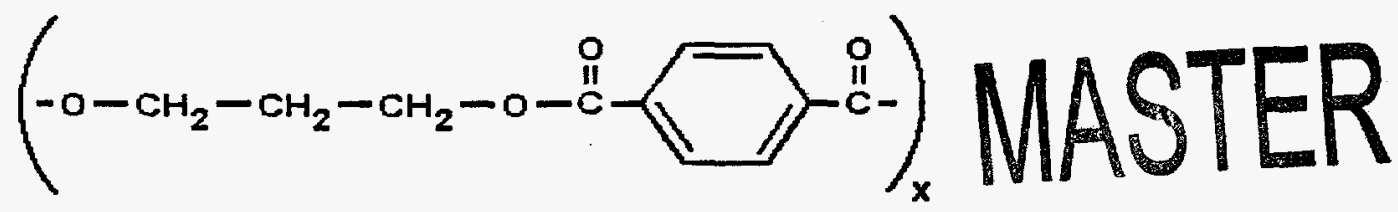
contractor of the U.S. Government under contract No. DE-AC05-96OR22464. Accondingly, the U.S. Government retains a nonexclusive, royalty-free ticense to publish or reproduce the published form of this contribution, of allow others to do so, for U.S. Government purposes." 


\section{DISCLAIMER}

This report was prepared as an account of work sponsored by an agency of the United States Government. Neither the United States Government nor any agency thereof, nor any of their employees, make any warranty, express or implied, or assumes any legal liability or responsibility for the accuracy, completeness, or usefulness of any information, apparatus, product, or process disclosed, or represents that its use would not infringe privately owned rights. Reference herein to any specific commercial product, process, or service by trade name, trademark, manufacturer, or otherwise does not necessarily constitute or imply its endorsement, recommendation, or favoring by the United States Government or any agency thereof. The views and opinions of authors expressed herein do not necessarily state or reflect those of the United States Government or any agency thereof. 


\section{DISCLAMIER}

Portions of this document may be illegible in electronic image prodnets. Imsges are produced from the best avaliable origion docomentert 
Because PTT is a relative new polymeric material, only limited information is available about its thermal properties. Its equilibrium melting temperature has been estimated to be about $510 \mathrm{~K}$ and a glass transition temperature between 315 and $345 \mathrm{~K}$ was reported, dependent on thermal pretreatment $[1,2]$. No heat capacities of PTT have been reported earlier. Poly(ethylene terephthalate) and (PBT), in contrast, have been characterized in our laboratory [3]. The thermodynamic properties of PET and PBT, such as their heat capacity, $\mathrm{Cp}$, enthalpy, $\mathrm{H}$, entropy, $\mathrm{S}$, and free enthalpy, $G$, and the transition behavior have been collected in the ATHAS data bank, using all prior work, published over the last 40 years [4].

In this paper, a first step of a quantitatively study of the heat capacity of PTT is described, using differential thermal analysis (DSC) and temperature-modulated DSC (TMDSC) for measurement and the ATHAS Scheme for data analysis. The measured heat capacity of solid PPT has been linked to the vibrational motion of solid of PTT using an optimization which yields the characteristic temperatures $\Theta_{1}$ and $\Theta_{3}$ as of a Tarasov equation [5,6]. The heat capacity of liquid PTT was compared to data obtained from the ATHAS addition scheme. Knowing the vibrational heat capacity and the linear extrapolation of the liquid from the melt as base-lines, the the heat of fusion $\left(\Delta \mathrm{H}_{\mathrm{f}}\right)$ and the change of heat capacity, $\Delta \mathrm{C}_{\mathrm{p}}$, at the glass transition temperature, $T_{g}$, could be estimated for semicrystalline and amorphous samples. Also, the crystallinity, $w_{c}$, the mobile amorphous fraction, $w_{a}$, and the rigid-amorphous fraction, $w_{r}$, have been determined. Extended results and discussions will be presented in the full paper [7].

\section{Calculation of the Heat Capacity for the Solid and Liquid States}

The computation of the heat capacity of solid PTT is based on an approximate group vibrational spectrum and the Tarasov approach for the skeletal vibrations, using the well-established ATHAS scheme [8]. In short, the experimental heat capacities $C_{p}(\exp )$ at constant pressure are first converted to heat capacities at constant volume $C_{\mathrm{V}}(\exp )$ using the Nernst-Lindemann approximation $[9,10]$. Assuming that $\mathrm{C}_{\mathrm{v}}(\exp )$ contains at sufficiently low temperatures only vibrational contributions, the experimental heat capacity at constant volume is then separated into the heat capacities linked to the group and skeletal vibrations. The latter are fitted to the Tarasov function as outlined in Ref. [5] to obtain two characteristic parameters $\Theta_{1}$ and $\Theta_{3}$, where $\Theta=h v / k$ and represents the frequency in kelvin; $h$ and $k$ are Planck's and Boltzman's constants, respectively. The heat capacities from the group vibrations were estimated from the experimental infrared and Raman frequencies or full normal mode calculations of the macromolecules, and are represented as single Einstein frequencies or box-distributions [8]. Knowing the values of $\Theta_{1}$ and $\Theta_{3}$ from a best fit of the experimental, skeletal heat capacity and the list of group vibrations, one can calculate the heat capacity at constant volume $C_{\mathrm{v}}(\mathrm{cal})$ and then convert again to the calculated heat capacity at constant pressure $\mathrm{C}_{\mathrm{p}} \mathrm{s}(\mathrm{cal})$. The heat capacity can be calculated for a wide temperature range $(0.1$ to $1000 \mathrm{~K})$ and serve as a 
baseline of the vibrational heat capacity. Any change in motion, as caused by the excitation of large-amplitude motion (translation, rotation, or conformational motion), as well as gradual absorption or evolution of latent heats can be judged against this baseline.

The heat capacity of the liquid state $\left(\mathrm{C}_{\mathrm{p}}{ }^{\mathrm{L}}\right)$ is more difficult to link to molecular motion than for the solid state. There is no adequate and simple microscopic theory of liquids. Empirically, it was found however, that $\mathrm{C}_{\mathrm{p}}{ }^{\mathrm{L}}$ is usually a linear function of temperature. Using an addition scheme developed for ATHAS, the heat capacity of liquid PTT can be obtained from contributions of its structural groups.

\section{Experimental Details}

Poly(trimethylene terephthalate) was supplied by Shell Chemical Company and analyzed after various thermal pretreatments. To obtain a sample that is as amorphous as possible, the PTT was heated above its melting temperature and quenched outside the calorimeter in liquid nitrogen. Semicrystalline PTT of different crystallinity were made by cooling of the sample in the calorimeter to below the glass transition with rates between 1 and $30 \mathrm{~K} / \mathrm{min}$. For transition measurements by standard DSC and for heat capacity by TMDSC, 2-6 mg of sample were used. For measurements of heat capacity by standard DSC 20-30 mg of sample were needed to gain highest precision. Standard sapphire $\left(\mathrm{Al}_{2} \mathrm{O}_{3}\right)$ was used for heat capacity calibration at each temperature for both, standard and temperaturemodulated DSC. The temperature calibration was done at the onsets of melting transition peaks of several standards.

Two different instruments were used for analysis of the transition behavior and measurement of he:: capacities: A DSC 820 from Mettler Toledo Inc., for standard DSC; and an MDSCTM 2920 of TA Instruments, Inc. for quasi-isothermal measurements by TMDSC.

Quasi-isothermal measurements of heat capacity with TMDSC have been described earlier in detail $[11,12]$. Three quasi-isothermal runs (sample, reference, and asymmetry calibration) were carried out with a modulation period, $p$, of $60 \mathrm{~s}$, and a sample-temperature amplitude, $\mathrm{A}$, of $0.5 \mathrm{~K}$. The reversing heat flow amplitudes were of the three runs were combined in the standard method to establish heat capacity [5]. The base temperature was increased in steps of 10 or $2 \mathrm{~K}$, depending on the degree of change of the heat capacity.

The measurement of heat capacity by standard DSC was carried out at a heating rate of $10 \mathrm{~K} / \mathrm{min}$. As in TMDSC, three runs were carried out and analyzed in the standard fashion, tabulated in $10 \mathrm{~K}$ intervals.

\section{Results and Discussion}

The measured heat capacities of solid, liquid, and semicrystalline PTT are presented in Figure 1. The figure shows a typical example of standard DSC from 190 to 570 
K. Figure 2 shows a comparison of the same sample with data by quasi-isothermal TMDSC from 223 to $553 \mathrm{~K}$. Also drawn in Figs. 1 and 2 are the calculated heat capacities as described above.

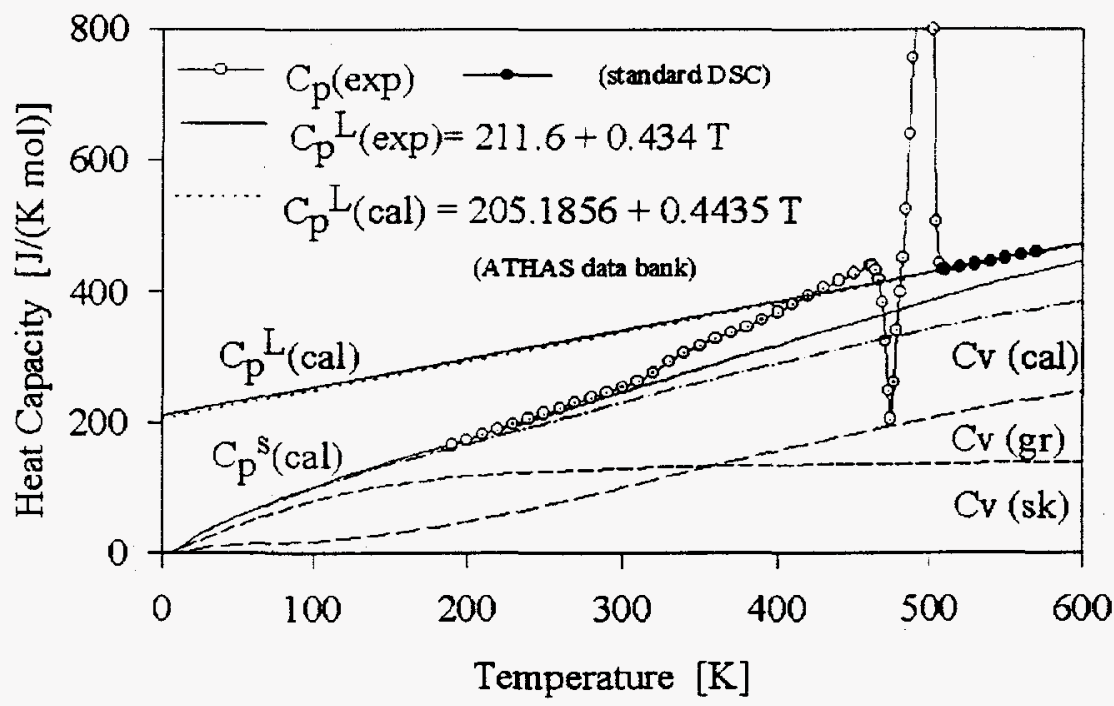

Figure 1. Experimental and calculated heat capacities of solid and liquid PTT.

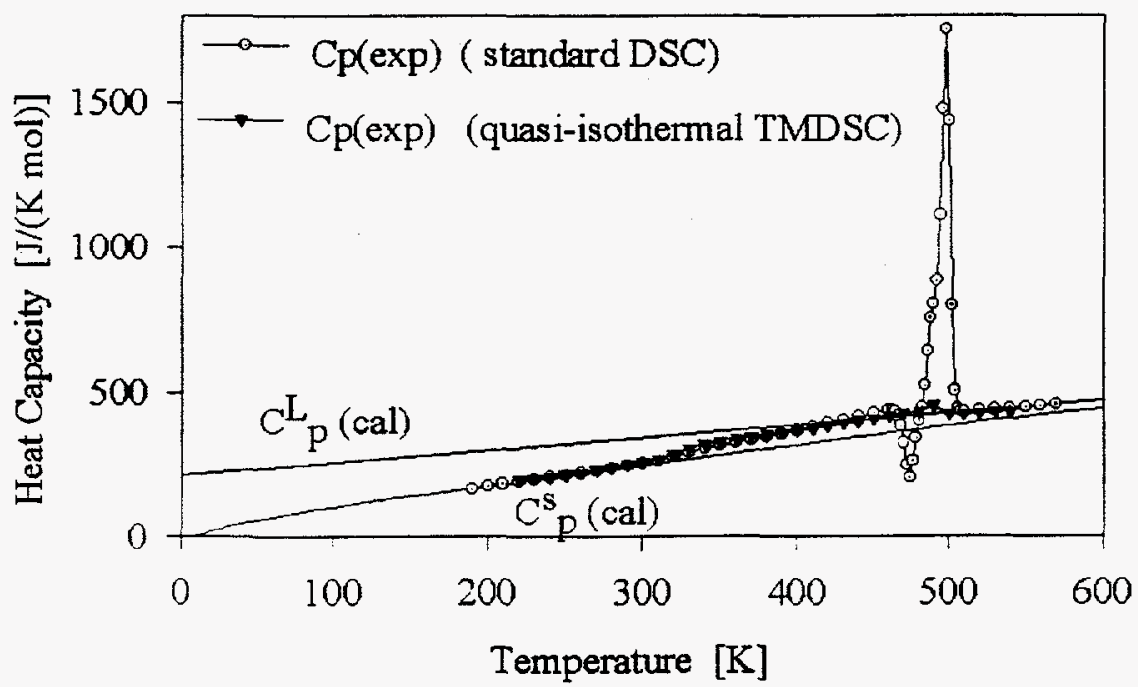

Figure 2. Heat capacity of PTT by standard DSC and quasi-isothermal TMDSC (reversing heat capacity). The solid lines indicate the heat capacities of $100 \%$ amorphous and $100 \%$ crystalline PTT. 
In order to evaluate the vibrational heat capacity of PTT, its 75 degrees of freedom of the 25 atoms of the repeating unit were separated into group vibrations $\left(\mathrm{N}_{\mathrm{gr}}=58\right)$ and skeletal vibrations $\left(\mathrm{N}_{\mathrm{sk}}=17\right)$. The approximate frequency spectra of the groups were adjusted from PET [3] by adding one $\mathrm{CH}_{2}$-group. Next, the experimental heat capacities for the different glassy and semicrystalline PTTs were averaged at each temperature from $190 \mathrm{~K}$ to $300 \mathrm{~K}$ (below $\mathrm{T}_{\mathrm{g}}$ ). After conversion to $\mathrm{C}_{\mathrm{V}}$, the group contributions were subtracted, as will be outlined in the full paper [7], to obtain the experimental, skeletal heat capacity. This, in turn, was fitted to the Tarasov equation, as described above. Since not enough data at low-temperature are available, the computation of the solid heat capacity was made using an estimated value of $\Theta_{3}=42 \mathrm{~K}$. The fitting resulted in $\Theta_{1}=542 \mathrm{~K}$. With these parameters the vibration-only heat capacity for solid PTT, $\mathrm{C}_{\mathrm{p}}{ }^{\mathrm{s}}(\mathrm{cal})$, was calculated from 0.1 to 1000 $\mathrm{K}$ as shown in Figs 1 and 2 . Within the range of measurement the experiment agrees with the calculations to better than $\pm 2 \%$. The measurements of the heat capacities for the liquid state above the melting temperature were fitted to a linear function. Figure 1 shows the experimental data (full circles ) and their extrapolation to low temperatures (solid line). Both agree well with the calculated heat capacity from the ATHAS addition scheme (dashed line, maximum error less than $\pm 0.5 \%$ ).

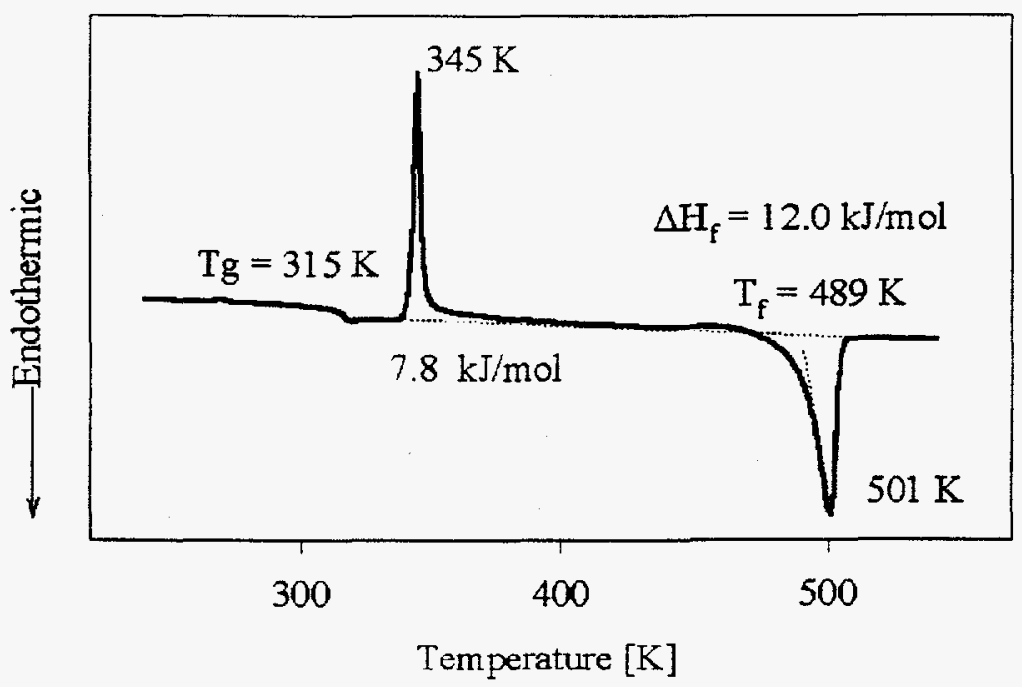

Figure 3. Standard DSC trace for quenched PTT.

Figure 2 shows the good agreement found between the heat capacities of solid and liquid PTT obtained by standard and temperature-modulated DSC. Major differences in $\mathrm{Cp}(\exp )$ are, however, found in the transition region as will be discussed in the full paper [7]. 
The glass transition temperature, $T_{g}$ of the semicrystalline PTT occurs at 331 $\mathrm{K}$ with a $\Delta \mathrm{C}_{\mathrm{p}}$ of $38 \mathrm{~J} /(\mathrm{K} \mathrm{mol})$ (see Fig. 2, temperature of half-devitrification). With different thermal histories, $T_{\mathrm{g}}$ varied from $315-345 \mathrm{~K}$. Figure 3 shows heat-flow data of a sample of low crystallinity with a $T_{g}$ of $315 \mathrm{~K}$. Typical for such largely amorphous samples is the sharp crystallization exotherm at $\approx 345 \mathrm{~K}$. The semicrystalline PTT of Fig. 1 shows a much smaller ordering exotherm at $460-480 \mathrm{~K}$. The melting endotherm in Fig. 3 occurs between 480 and $505 \mathrm{~K}$ with an onset temperature $T_{\mathrm{f}}=489 \mathrm{~K}$. For Fig. 1 the measured heat of fusion is $13.8 \mathrm{~kJ} / \mathrm{mol}$.

To calculate the change of crystallinity with temperature, use was made of the following equation:

$$
C_{\mathrm{p}}{ }^{*}(\exp )=w_{\mathrm{c}} C_{\mathrm{p}}(\text { solid })+\left(1-w_{\mathrm{o}}\right) C_{\mathrm{p}} \text { (liquid) }-\frac{\mathrm{d} w_{\mathrm{c}}}{\mathrm{d} T} \Delta H_{\mathrm{f}}(T)
$$

where $\mathrm{C}_{\mathrm{p}}{ }^{*}(\exp )$ is the experimental heat capacity, $\mathrm{Cp}$ (solid) and $\mathrm{Cp}$ (liquid) are the calculated crystalline and melt heat capacities, $w_{c}$ is the temperature-dependent crystallinity, and $\Delta \mathrm{H}_{\mathrm{f}}$ is the, also temperature-dependent, heat of fusion. The results are presented in Fig. 4 for the same sample as shown in Figs. 1 and 2.

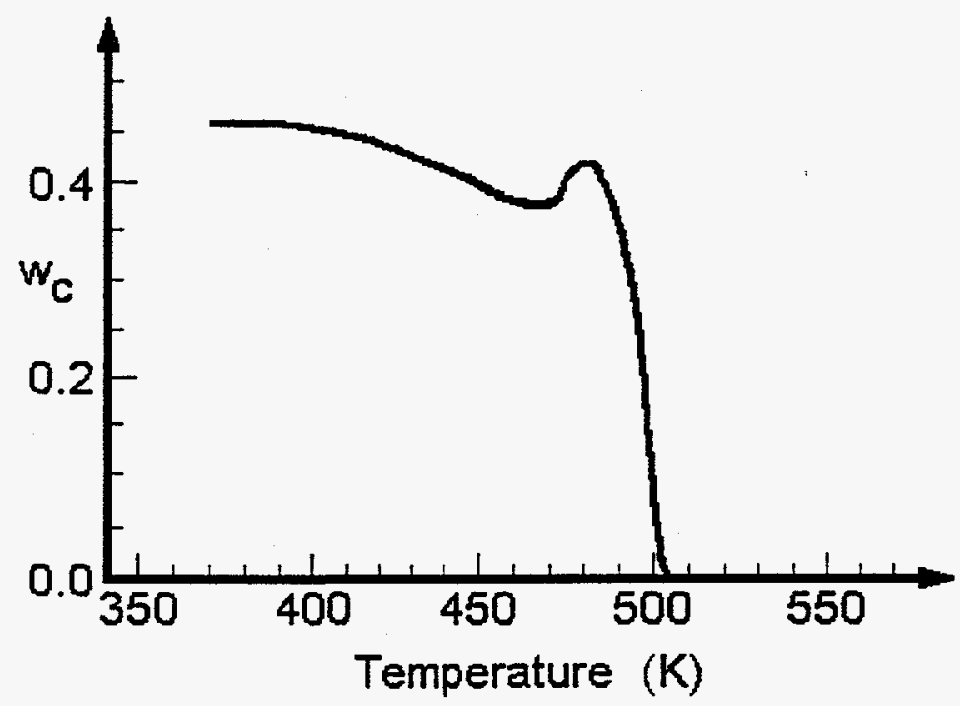

Figure 4. Plot of the dependence of crystallinity on temperature according to Eq. (1).

The changes of $\Delta \mathrm{C}_{\mathrm{p}}$ at $\mathrm{T}_{\mathrm{g}}$ and $\Delta \mathrm{H}_{\mathrm{f}}$ for samples with different thermal history are presented in Fig. 5. Extrapolation (solid line) to $100 \%$ crystalline PTT gives the equilibrium heat of fusion, $30 \mathrm{~kJ} / \mathrm{mol}$ [entropy of fusion of $58.8 \mathrm{~J} /(\mathrm{K} \mathrm{mol})$ ]. The experimental data show occasional deviations from linearity which may suggest a rigid amorphous fraction, indicated by a too low heat of fusion when compared to 
the $\Delta \mathrm{C}_{\mathrm{p}}$. The rigid-amorphous fraction can be calculated from $\mathrm{w}_{\mathrm{r}}=1-\mathrm{w}_{\mathrm{a}}-\mathrm{w}_{\mathrm{c}}$ where:

$$
w_{\mathrm{r}}=1-\frac{\left[C_{\mathrm{p}}^{*}(\exp )-C_{\mathrm{p}}(\text { solid })\right]}{\left[C_{\mathrm{p}}(\text { liquid })-C_{\mathrm{p}}(\text { solid })\right]}-w_{\mathrm{c}}
$$

Knowing $\mathrm{C}_{\mathrm{p}}$ of the solid, liquid and the transition parameters, the thermodynamic functions: enthalpy $\mathrm{H}$, entropy $\mathrm{S}$ and Gibbs function $\mathrm{G}$ can be calculated as a function of temperature and will be presented in full paper [7].

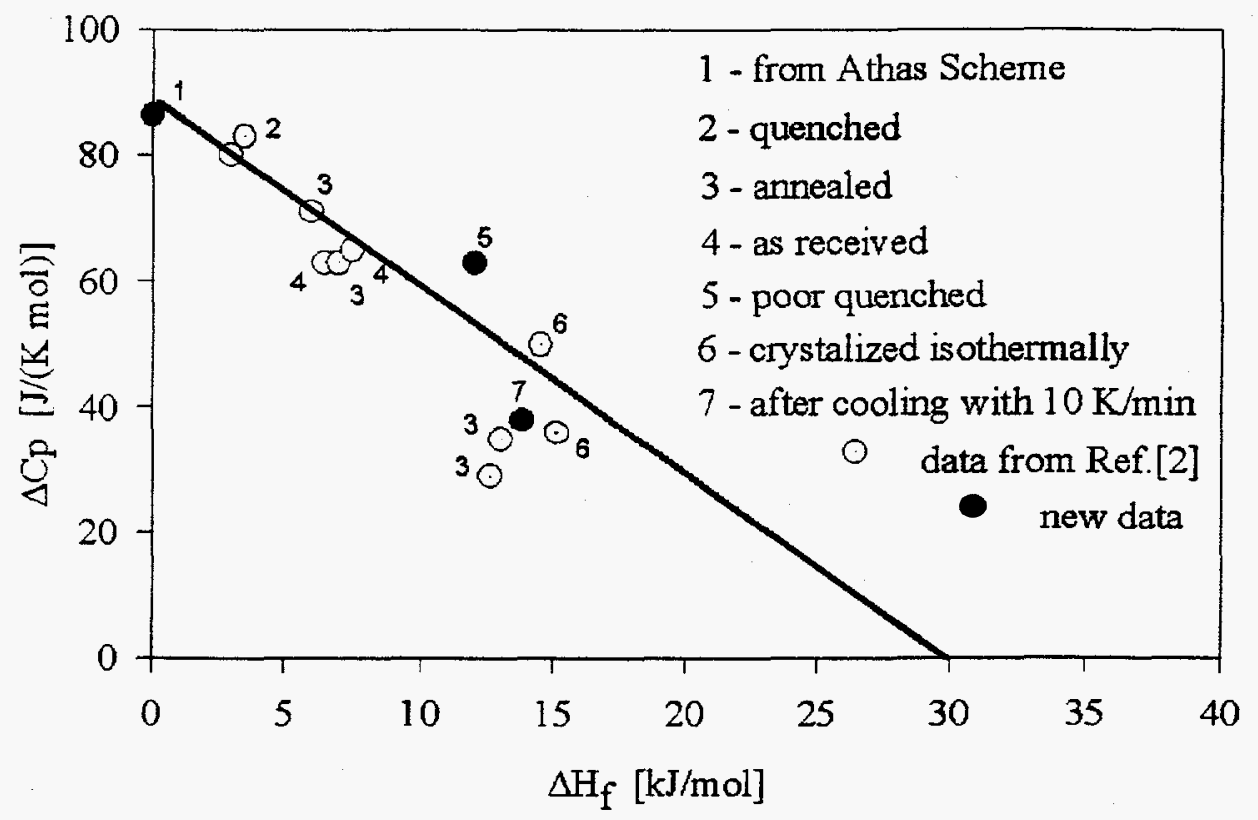

Figure 5. The plot of $\Delta C_{p}$ at Tg vs $\Delta H_{f}$ for samples of PTT with different crystallinities.

To further separate possible rigid amorphous fractions and early melting the TMDSC measurements were performed as shown in Fig. 2. First one can see from the comparison with standard DSC that both the crystallization at about $470 \mathrm{~K}$ and the melting endotherm at about $409 \mathrm{~K}$ are practically fully irreversible and do not show in TMDSC. Next, one can see practically coinciding DSC and TMDSC traces from 220 to $450 \mathrm{~K}$. This shows that in this sample there is no significant rigidamorphous fraction, as is also indicated in Fig. 5.

Further discussion of the samples below the straight line of Fig. 5 will be given in Ref. [7]. The annealing experiments illustrated in Fig. (5) were done in the calorimeter by heating for $30 \mathrm{~s}$ to various temperatures from 328 to $403 \mathrm{~K}$, followed by cooling to $223 \mathrm{~K}$ for subsequent analysis. The isothermal crystallizations were 
performed between 443 and $483 \mathrm{~K}$ on cooling from the melt. These samples were also first cooled to $223 \mathrm{~K}$ before analysis.

It will be of interest to find reasons for the high values of the reversing heat capacity at the low-temperature side of the melting peak. This observation was also made for several other polymers analyzed with quasi-isothermal TMDSC. The level of $\mathrm{C}_{\mathrm{p}}$ reaches that of the liquid $\mathrm{C}_{\mathrm{p}}$. More experimental evidence from TMDSC in the beginning melting region must be collected to see if this extra thermal energy is a heat-capacity contribution or a latent heat resulting from premelting.

In initial solid-state NMR reports [2] it was stated that the spin relaxation times could not be fitted to a two-phase model and had to involve a third, rigid amorphous phase. Also, it will be useful to find a possible link of this observation to X-ray data on the crystal structure, also in progress. The main problem that seems to show is that samples of different thermal history are sufficiently changed that data comparison is only possible on identical samples.

\section{Acknowledgments}

This work was financially supported by the Div. of Materials Res., NSF, Polymers Program, Grant \# DMR-9703692 and Oak Ridge National Laboratory, managed by Lockheed Martin Energy Research Corp. for the U.S. Department of Energy, under contract number DE-AC05-9 6OR22464. Support for instrumentation came from TA Instruments, Inc. and Mettler-Toledo.

\section{References}

1 C. C. Gonzales, J. M. Perena and A. Bello, J. Polym. Sci. Part. B: Polymer Phys., 26 (1988) 1397.

2 J. Grebowicz, H. H. Chuah, Progress Report., Shell, (1996).

3 S. Z. D Cheng, R. Pan, H. S. Bu, M.-Y. Cao and B. Wunderlich, Makromol. Chem. 189, 1579 (1988); S. Z. Cheng, S. Lim, L. H. Judovits, and B. Wunderlich, Polymer, 28, 10 (1987).

4 ATHAS Data Bank, \#250, \#271; WWW address on the Internet: http://funnelweb.utcc. utk.edu/ athas.

5 B. Wunderlich, "Thermal Analysis." Academic Press, Boston, 1990.

6 V. V. Tarasov, Zh. Fiz. Khim., 24 (1950) 111.

7 M. Pyda and B. Wunderlich, J. Thermal Analysis in preparation (1997).

$8 \quad$ B. Wunderlich, Pure and Applied Chem., 67 (1995) 1919.

9 W. Nernst and F. A.Lindemann, Z. Electrochem., 17 (1911) 817.

10 R. Pan, M. Varma-Nair, and B. Wunderlich, J. Thermal Analysis, 35 (1989) 955.

11 B. Wunderlich, Y, Jin, and A. Boller, Thermochim. Acta, 238 (1994) 277.

12 A. Boller, Y. Jin, and B. Wunderlich J. Thermal Analysis, 42 (1994) 307. 